\title{
An On-Line Classification Approach of Visitors' Movements in 3D Virtual Museums
}

\author{
Kingkarn Sookhanaphibarn and Ruck Thawonmas \\ Intelligent Computer Entertainment Laboratory \\ Department of Human and Computer Intelligence \\ Ritsumeikan University \\ Kusatsu, Shiga 525-8577, Japan
}

\begin{abstract}
Recommender systems, in virtual museums and art galleries, providing the personalization and context awareness features require the off-line synthesis of visitors' behaviors therein and the off-line training stage of those synthetic data. This paper deals with the simulation of four visitors' styles, i.e., ant, fish, grasshopper, and butterfly, and the classification of those four styles using an Adaptive Neuro-Fuzzy Inference System (ANFIS). First, we analyze visitors' behaviors related to a visit time and an observation distance. Then, the proposed synthesis procedure is developed and used in the off-line training stage of ANFIS. The training and testing data are the average and variance of a set of visitors' attention data computing by the proposed function of the visit time and observation distance variables. Therefore, the trained ANFIS can identify the behavior style of an on-line visitor using the training set of synthetic data and its memberships can describe degrees of uncertainty in behavior styles.
\end{abstract}

Keywords: 3D virtual environment; Recommender system; Visitor movement; Visitor behavior; Visualization; Adaptive Neuro-Fuzzy Inference Systems (ANFIS).

\section{Introduction}

Currently, the number of existing virtual museums in Second Life (SL) is greater than 150 locations, characteristics of which vary tremendously in terms of scale and size, artifact types and exhibitions, media technologies, and target visitors, as reported by Urban [1. An immersively interactive feature embedded in SL can attract the attention of up to 30 official organizations existing in Real Life (RL). One of them is the Smithsonian Latino Center opened the Smithsonian Latino Virtual Museum (LVM) in March 19, 2009 which provides the vast and rich collections, research and scholarship, exhibitions and educational activities related to U.S. Latinos and Latin America. This trend leads to the need of a conceptual framework of digital museums in the $3 \mathrm{D}$ virtual world, which was designed, developed and evaluated in [2]3].

Recommender modules, such as, guide avatar and personal route generation modules, were designed in the conceptual framework proposed by Sookhanaphibarn 
and Thawonmas 3] to achieve high visitors' satisfaction. The guide avatar addressed in 4] can create a new text for an individual visitor, at each time when he/she views an object. The guide avatar never repeats information which it has already expressed. For example, if a visitor moves from one artifact to another artifact, both of which belong in the classical period; the guide avatar will not repeat the description on classical period at the latter location. A personal route for a museum tour assists visitors in making a decision where they should stop over at and how long they should take. Since visitors in SL can teleport to any place with a constantly short time, the personal route generation module for a museum tour is a novel concept in the personalization issue.

Visitors in an art gallery have their stereotypical movement, which is categorized in four styles as proposed by Veron and Levasseur [5]. Four visiting styles based on an animals' behavior metaphor are ant, fish, grasshopper, and butterfly styles. The ant visitors spend quite a long time to observe all exhibits by walking closer to exhibits but avoids empty spaces. The fish visitors prefer to move to and stop over at empty spaces but avoid areas near exhibits. The grasshopper visitors spend a long time to see selected exhibits but ignore the rest of exhibits. The butterfly visitors observe all exhibits but spend varied times to observe each exhibit. Identifying their visiting styles can take advantage of recommender systems in virtual museums as mentioned in [6/789].

In this paper, we propose an on-line classification system of four visitor styles based on their definitions, which are summarized in 510]. This is based on our hypothesis that a visiting style can be simulated by using mathematic functions. To validate our hypothesis, we conduct an adaptive neuro-fuzzy inference system (ANFIS) trained by the synthetic visitor data in the off-line stage and tested by the new visitor data in the on-line stage. ANFIS is a hybrid learning algorithm to identify the membership function parameters of four visiting styles. The contributions of this work are (a) the novel simulation method of visiting styles in art galleries and museums in SL, (b) the proposed function to measure the visitors' attention of each exhibit, (c) the classification using ANFIS based on the average and variance of a set of visitors' attention data of all exhibit, and (d) our implications to applications of these findings.

\section{Four Visiting Styles}

\subsection{Definition}

Chittaro and Ieronutti 10 described four visiting styles based on results from their visualization tool, where black highlights areas more traveled, white identifies the areas less traveled and different shades of gray are used to identify intermediate previous situations, as shown in Table 1. Corresponding to four visiting styles, the aforementioned researches [5]7,10] described them beneficial to a physical environment design in museums as shown in Table 1. Note that Fig. 1] shows the visualization of a visitor in the art gallery, where all exhibits are placed on the wall. Without loss of generality, an art gallery shown in Fig. 2 is used as the museum map in our synthetic data with 12 exhibits hanging in three sides of the room. 
Table 1. Visualization and recommended environment design of four visiting styles

\begin{tabular}{|c|c|c|}
\hline Styles & Visualization & Recommendation \\
\hline Ant & $\begin{array}{l}\text { The center of most space on the map is colored } \\
\text { in black, and there are no large differences in } \\
\text { the time different exhibits have been seen as } \\
\text { shown in Fig. [1 (a). }\end{array}$ & $\begin{array}{l}\text { They need to be guided by a powerful } \\
\text { rationale and this is the reason why } \\
\text { they usually follow the path proposed } \\
\text { by the museum curator. }\end{array}$ \\
\hline Fish & $\begin{array}{l}\text { The areas near all exhibits on the map are } \\
\text { colored in black as shown in Fig. 1 (b). }\end{array}$ & \begin{tabular}{|l} 
They prefer a holistic observation \\
area, such as the center of the room.
\end{tabular} \\
\hline Grasshopper & $\begin{array}{l}\text { The areas near some exhibits are colored with } \\
\text { highly variable shades of gray due to the fact } \\
\text { that this visitor spends a variable time to ob- } \\
\text { serve different exhibits and ignores the rest of } \\
\text { them as shown in Fig. 1 } 1 \text { (c). }\end{array}$ & $\begin{array}{l}\text { They move directly to their selective } \\
\text { exhibits. }\end{array}$ \\
\hline Butterfly & $\begin{array}{l}\text { The areas near all exhibits are colored with } \\
\text { different shades of gray and some are colored } \\
\text { with black, but less regularly than those of the } \\
\text { ant visitors as shown in Fig. [1 (d). }\end{array}$ & $\begin{array}{l}\text { They refuse to follow the given path } \\
\text { designed by other people and prefer } \\
\text { their own route. }\end{array}$ \\
\hline
\end{tabular}

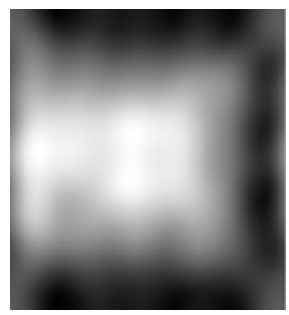

(a) Ant style

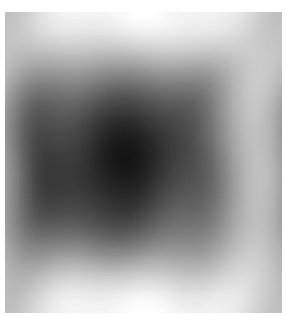

(b) Fish style

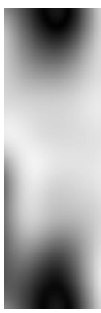

(c) Grasshopper style

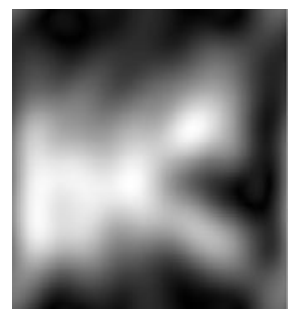

(d) Butterfly style

Fig. 1. Visualization of visitor styles

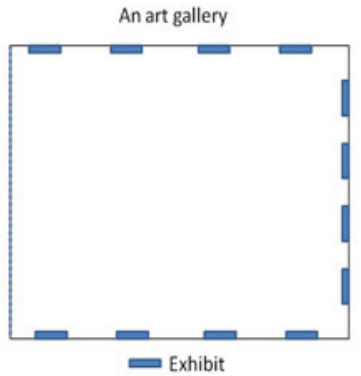

Fig. 2. The plan of an art gallery which all exhibits are hung on the wall

\subsection{Algorithms of Data Synthesis}

To synthesize the visit map, there are two input data as follows: a plan of museum, which illustrates the location of all exhibits, and his/her visitor type. We propose a synthesis approach for four visiting styles through the following steps: 
1. Selective exhibit randomness

2. Preference weight randomness

3. Visit time distribution

4. Noise synthesis

5. Smoothing filter

There are four proposed synthesis procedures, i.e., ant, fish, grasshopper, and butterfly styles as described in Fig. 3 and their notations as defined in Table 2, All four synthesis procedures have one input argument, $H$ or a set of exhibits, and one output, $V$ or a visit map. Our synthetic visit maps have darkest colored pixels equal to 1 and brightest colored pixels equal to 0 . The difference between the grasshopper and the butterfly styles is the number of stopovers at exhibits, i.e., the former stops fewer than the latter does. The selective exhibit randomness step is used in these two visiting styles using a $\beta$ probability density function since two parameter $\gamma$ and $\beta$ can control the PDF curve corresponding to the grasshopper and butterfly paradigm of the number of stopovers. The $\beta$ PDF of a sample $x$ is written below:

$$
f(x ; \gamma, \beta)=\frac{1}{B(\gamma, \beta)} x^{\gamma-1} 1-x^{\beta-1} x,
$$

where $B(\cdot)$ is the Beta function as defined in [1]. In cases of both ant and fish styles, the selective exhibit randomness are not applied because the ant visitor stops at all exhibits and moves following the exhibit path proposed by a museum curator and the fish visitor stops at none of exhibits and moves only to the center of the room.

The preference weight randomness step generates varying stopover times at exhibits. Both ant and fish styles show no significant difference among their preferences of exhibits. Second, the visit time distribution functions are different among visiting styles. Third, noise synthesis is conducted using a Gaussian PDF. The implication of noise is randomly short stops in the exhibition area. Then, the smooth filtering such as a low pass filter is applied in order to discard the sharp detail and remove the noise. An example of the ant, fish, grasshopper, and butterfly visit maps derived by using the procedure is shown in Fig. 1] (a)-(d), respectively.

The aforementioned synthesis procedures require to construct a Voronoi partition of the set of pixels in a museum area. This is a division of the museum area into regions around each exhibit; a region is the set of pixels that are closer to that exhibit than to any other. Let $\mathbf{h}_{k}$ be the position of exhibit $k$ and let $H=\left\{\mathbf{h}_{k} \mid 1 \leq k \leq M\right\}$ be a set of all positions of exhibits, where $M$ is a total exhibit. Let $\mathbf{u}$ be the position of a visitor. The exhibit $\mathbf{h}_{k^{*}}$ nearest to a visitor $\mathbf{u}$ is calculated by the following

$$
k^{*}=\operatorname{argmin}_{k}\left\|\mathbf{h}_{k}-\mathbf{u}\right\| \quad \text { where } 1 \leq k \leq M
$$

where $\left\|\mathbf{h}_{k}-\mathbf{u}\right\|$ is the Euclidean distance between exhibit $\mathbf{h}_{k}$ and visitor $\mathbf{u}$. 
Table 2. Notations used in the synthesis procedures of four visiting styles

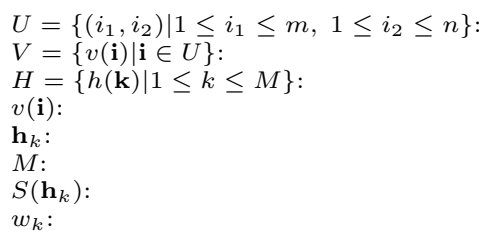

The set of pixels in a museum area

Visit map

A set of all positions of exhibits

The visitor's stopover time at pixel i, called Visit time

The position of exhibit $k$

A total exhibit

A set of pixels $\mathbf{i}$ belonging to $\mathbf{h}_{k}$

The preference weight of exhibit $\mathbf{h}_{k}$

\section{Classification System of Visitors' Behaviors in 3D Virtual Museums}

\subsection{Proposed Function of Visitor's Attention at Each Exhibit}

Considering the visualization scheme of visiting styles in 10[12, we can discard the temporal information in trajectories data of visitor movement in a museum. Given a visit map, $V$, it is obvious that a dark colored area is a key role to identify the visiting styles because it illustrates a long stopover. However, the dark colored area near or far from an exhibit is considered as a parameter to measure the visitors' attention to the exhibit. Given a museum area, $U$, the observation distance and map are defined as follows.

1. Observation distance $o(\mathbf{i})$ is defined as the visitor's fuzzy distance from the nearest exhibit

2. Observation map is defined as a set of $o(\mathbf{i})$, where $\mathbf{i} \in U$

To compute the observation distance, the observation distance function $\Psi\left(\mathbf{h}_{k}, \mathbf{u}\right)$ is defined as an exponential function of the distance from an exhibit $\mathbf{h}_{k}$ to a visitor $\mathbf{u}$ as follows:

$$
\Psi\left(\mathbf{h}_{k}, \mathbf{u}\right)=e^{-\rho\left\|\mathbf{h}_{k}-\mathbf{u}\right\|^{2}}
$$

$\rho$ is a weighted constant. In the same manner of an Gaussian-based activation function, this weighted constant is obtained from the variance of the Euclidean distance between exhibit $\mathbf{h}_{k}$ and pixel $\mathbf{i}$, for all $\mathbf{h}_{k}$ and $\mathbf{i}$, as shown in Eq.(4).

$$
\begin{gathered}
\mu=\operatorname{mean}\left\{\bigcup_{\mathbf{h}_{k} \in H} \wedge \mathbf{i} \in U\right. \\
\sigma^{2}=\operatorname{mean}\left\{\bigcup_{\mathbf{h}_{k} \in H} \wedge \mathbf{i} \in U\right. \\
\rho \\
\rho
\end{gathered}
$$

Fig. 4 shows a graph plotting of $\Psi\left(\mathbf{h}_{i}, \mathbf{u}\right)$ and $\left\|\mathbf{h}_{k}-\mathbf{v}\right\|$ where $\sigma^{2}$ is 0.07 . The observation distance is calculated as follows:

$$
o(\mathbf{i})=\Psi\left(\mathbf{h}_{k}, \mathbf{i}\right) \quad \text { where } \quad \mathbf{i} \in S\left(\mathbf{h}_{k}\right)
$$


Procedure: Synthesis of Ant visiting style

Input: $\quad H$

Output: $\quad V$

Step 1: Preference weight randomness is set by using the Gaussian PDF with the mean closer to 1 and smallest variance.

Step 2: Visit time $v(\mathbf{i})$ at pixel $\mathbf{i}$ is defined by using the following equation: $v(\mathbf{i})=w_{k} e^{-\rho\left\|\mathbf{h}_{k}-\mathbf{i}\right\|^{2}}$ where $\mathbf{i} \in S(\mathbf{k})$

Step 3: Smoothing filter is applied to the visit map obtained from Step 2.

Procedure: Synthesis of Fish visiting style

Input: $\quad H$

Output: $\quad V$
Step 1: Preference weight randomness is set by using the Gaussian PDF with the mean closer to 1

Step 2: Visit time $v(\mathbf{i})$ at pixel $\mathbf{i}$ is defined by using the following equation:

$v(\mathbf{i})=w_{k}\left(1-e^{-\rho\left\|\mathbf{h}_{k}-\mathbf{i}\right\|^{2}}\right)$ where $\mathbf{i} \in S(\mathbf{k})$

Step 3: Smoothing filter is applied to the visit map obtained from Step 2.

Procedure: Synthesis of Grasshopper visiting style

Input: $\quad H$

Output: $\quad V$

Step 1: Selective exhibit randomness is decided by using Beta PDF with $\gamma=1$ and $\beta=5$.

$P(\eta)=\frac{\eta^{\alpha-1}(1-\eta)^{\beta-1}}{\Gamma(\alpha) \Gamma(\beta)} \Gamma(\alpha+\beta)$

where $\Gamma$ is the gamma function and the parameters $\alpha$ and $\beta$ are related through

$\alpha=\mu \gamma$ and $\beta=(1-\mu) \gamma$ where $\gamma=\frac{\mu(1-\mu)}{\sigma}-1$.

The output of the PDF is the number of selected exhibits, as denoted by $L$.

Then, then selective exhibit is defined as a binary vector where $f_{i}$ is an element of the binary vector and $\sum_{i} f_{i}$ must be $L$.

Step 2: Preference weight randomness is set by using the Gaussian PDF with the mean closer to 1 and small variance.

Step 3: Visit time $v(\mathbf{i})$ at pixel $\mathbf{i}$ is defined by using the following equation:

$$
v(\mathbf{i})= \begin{cases}w_{k} e^{-\rho\left\|\mathbf{h}_{k}-\mathbf{i}\right\|^{2}} & \text { if } \mathbf{i} \in S(\mathbf{k}) \text { and } f_{k}=1 \\ 0 & \text { otherwise }\end{cases}
$$

Step 4: Noise synthesis is performed by using Gaussian PDF

Step 5: Smoothing filter is applied to the visit map obtained from Step 4.

Procedure: Synthesis of Butterfly visiting style

Input: $\quad H$

Output: $\quad V$

Step 1: Selective exhibit randomness is decided by using Beta PDF with $\gamma=5$ and $\beta=1$.

$P(\eta)=\frac{\eta^{\alpha-1}(1-\eta)^{\beta-1}}{\Gamma(\alpha) \Gamma(\beta)} \Gamma(\alpha+\beta)$

where $\Gamma$ is the gamma function and the parameters $\alpha$ and $\beta$ are related through

$\alpha=\mu \gamma$ and $\beta=(1-\mu) \gamma$ where $\gamma=\frac{\mu(1-\mu)}{\sigma}-1$.

The output of the PDF is the number of selected exhibits, as denoted by $L$.

Then, then selective exhibit is defined as a binary vector where $f_{i}$ is an element of the binary vector and $\sum_{i} f_{i}$ must be $L$.

Step 2: Preference weight randomness is set by using the Gaussian PDF with the mean closer to 1 and variance higher than the ant's.

Step 3: Visit time $v(\mathbf{i})$ at pixel $\mathbf{i}$ is defined by using the following equation:

$$
v(\mathbf{i})= \begin{cases}w_{k} e^{-\rho\left\|\mathbf{h}_{k}-\mathbf{i}\right\|^{2}} & \text { if } \mathbf{i} \in S(\mathbf{k}) \text { and } f_{k}=1 \\ 0 & \text { otherwise }\end{cases}
$$

Step 4: Noise synthesis is performed by using Gaussian PDF.

Step 5: Smoothing filter is applied to the visit map obtained from Step. 4.

Fig. 3. Synthesis procedures of four visiting styles

The visitor's attention at exhibit $\mathbf{h}_{k}$ is computed by using the visit time and the observation distance belonging to $S\left(\mathbf{h}_{k}\right)$, as shown below:

$$
A\left(\mathbf{h}_{k}\right)=\operatorname{mean}\left\{\bigcup_{\mathbf{i} \in S\left(\mathbf{h}_{k}\right)}\{o(\mathbf{i}) v(\mathbf{i})\}\right\}
$$

where $S\left(\mathbf{h}_{k}\right)$ is a set of pixels $\mathbf{i}$ belonging to $\mathbf{h}_{k}$, as defined by Eq.(2), and $o(\mathbf{i}) \geq \delta$. $\delta$ is a threshold in the range from 0 to 1 , which its implication is a observation distance limit. 


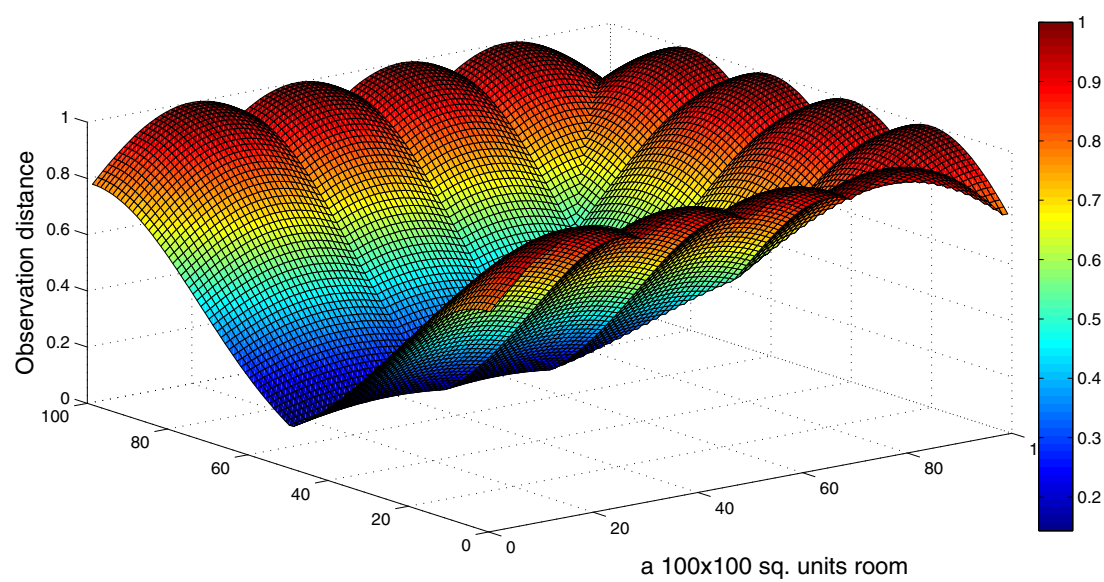

Fig. 4. Observation distance function of Eq. (3) where $H=\{(1,20),(1,40),(1,60)$, $(1,80),(20,100),(40,100),(60,100),(80,100),(100,20),(100,40),(100,60),(100,80)\}$

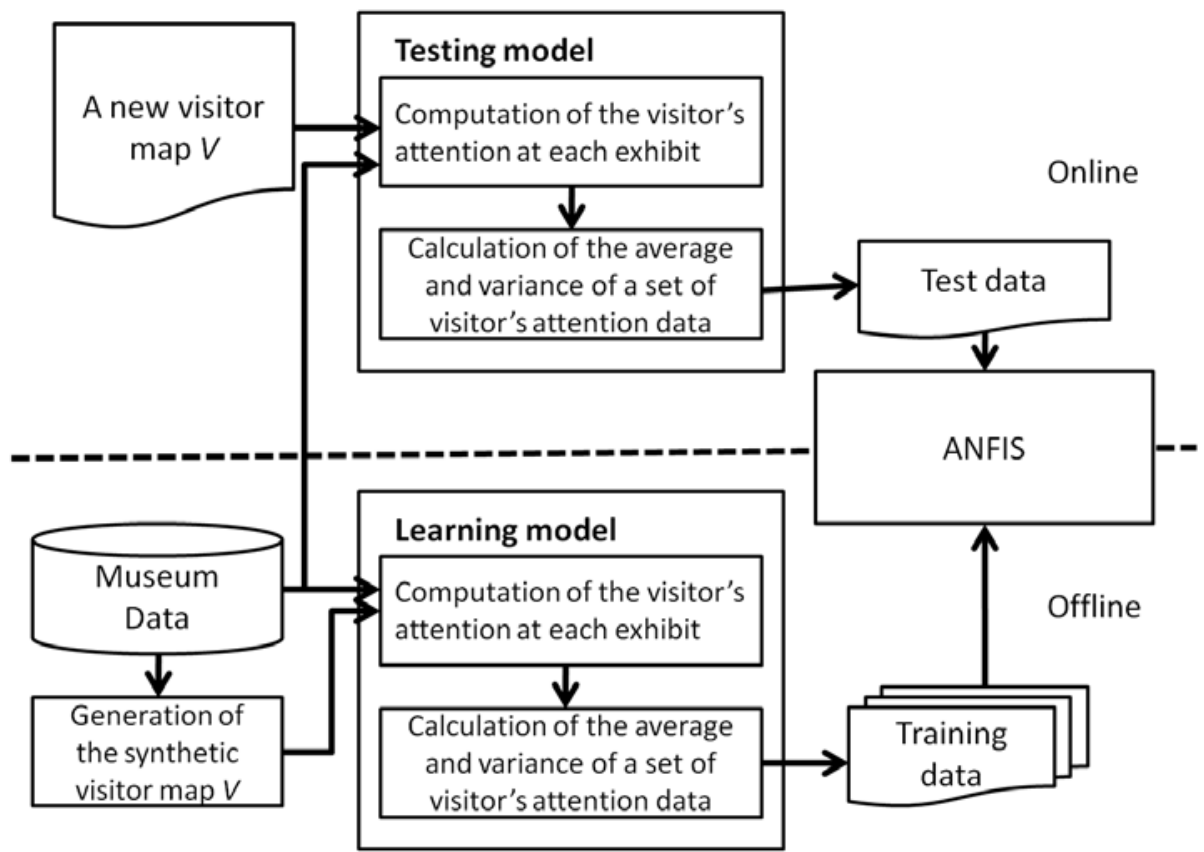

Fig. 5. Framework of the proposed classification system in off-line and on-line stages

\subsection{Framework of Classification System}

An overview of the proposed classification system is divided into on-line and off-line stages corresponding to learning and testing models, respectively. These models deal with the museum data, which is a set of the exhibits' positions in 


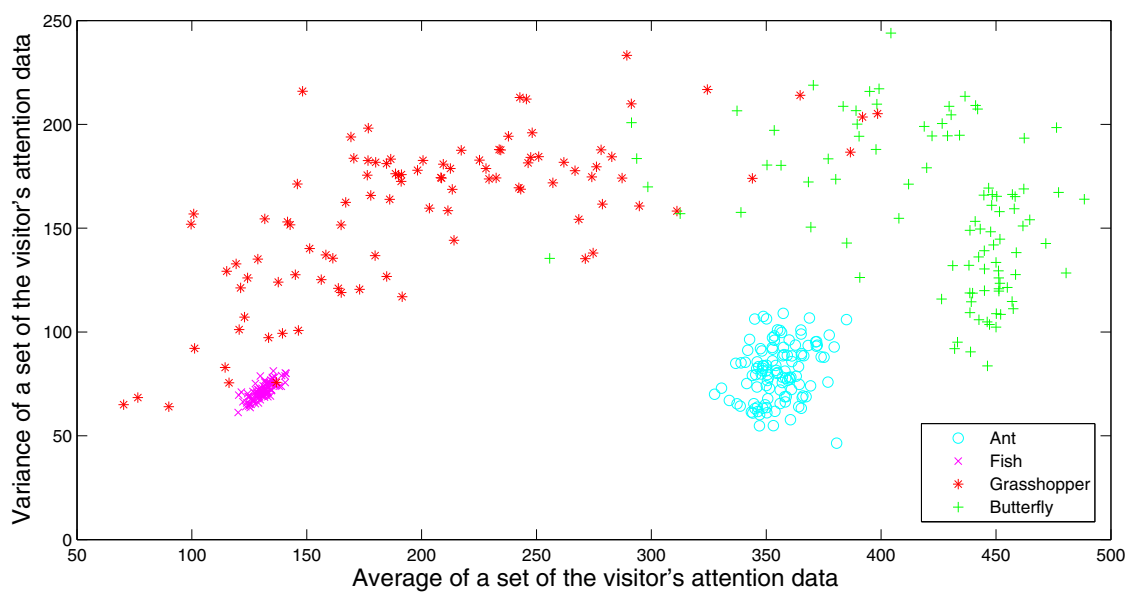

Fig. 6. 400 visitors of four clusters are plotted on the average and variance of their attention at all exhibits

a museum area, and the visit data, which is two dimensional time-series data. This framework performs the learning model by generating the virtual samples of visitors belonging to all four visiting styles as mentioned in Section 2.2. The virtual samples are represented by the visualization of a set of visitors' stopovertime data in the museum area, denoted by $V$ as described in Table 2 and Fig. 3.

The training and test data of ANFIS are the average and variance of a set of visitor's attention data, which the visitor's attentions $A\left(\mathbf{h}_{k}\right)$ for all $k$ are calculated by using Eq.(6). For example, there are 400 visitors each of which has the average and variance of their attentions as shown in Fig. 6. The clusters of four visitor types indicate that the clusters of ant and fish visitor types are isolated, which their probabilities found in [6] are 30\% and 20\%, respectively. The selective exhibit randomness is a cause of an overlapping area between grasshopper and butterfly clusters with the result that the Beta PDFs of both visitor types are a common area. However, the probability of grasshopper visitors found in the physical world is only $10 \%$ as reported in [6]. Therefore, a fuzzy inference system is used to classify the overlapped clusters and generate a linguistic rule-based classfier.

A fuzzy inference system is the process of formulating the mapping from a given input to an output using fuzzy logic. The mapping then provides a basis from which decisions can be made, or patterns discerned. ANFIS includes the neuro-adaptive learning method that works similarly to that of neural networks. The neuro-adaptive learning techniques provide a method for the fuzzy modeling procedure to learn information about a data set. The training data set is a set of input-output data, which the input data is the average and variance of a set of attention data obtained from the synthetic visit maps and the output is their visiting styles in the off-line stage, whereas the test data is from a new visitor in the on-line stage. 


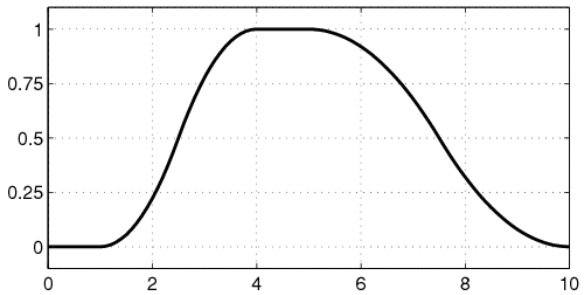

Fig. 7. Spline-curve-based membership function with the parameters $[a, b, c, d]=$ $[1,4,5,10]$

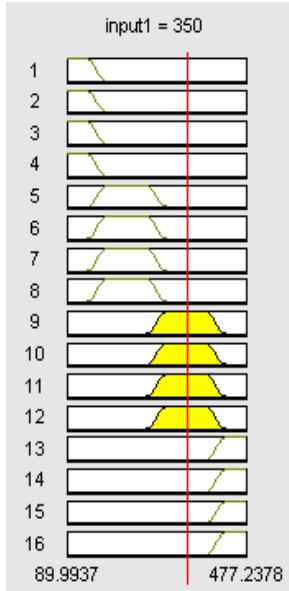

(a) Ant [349.90 81.22]

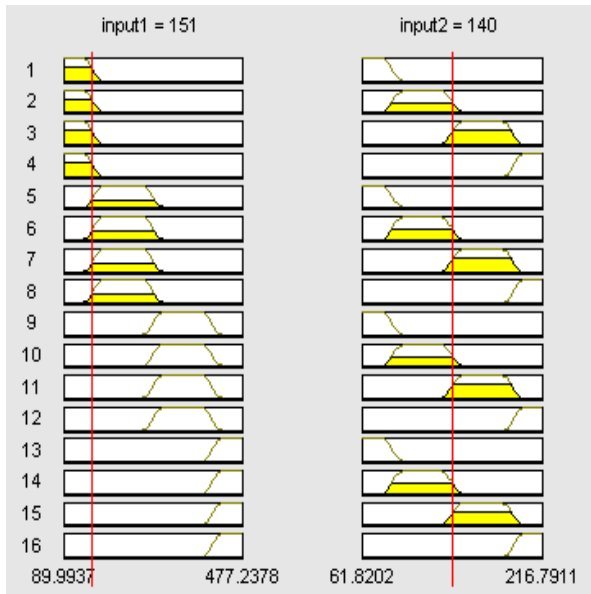

(c) Grasshopper [151.27 140.16]

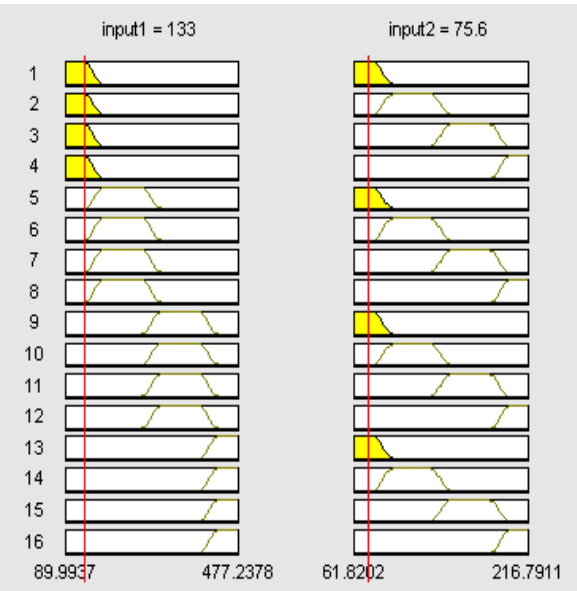

(b) Fish [132.80 75.63]

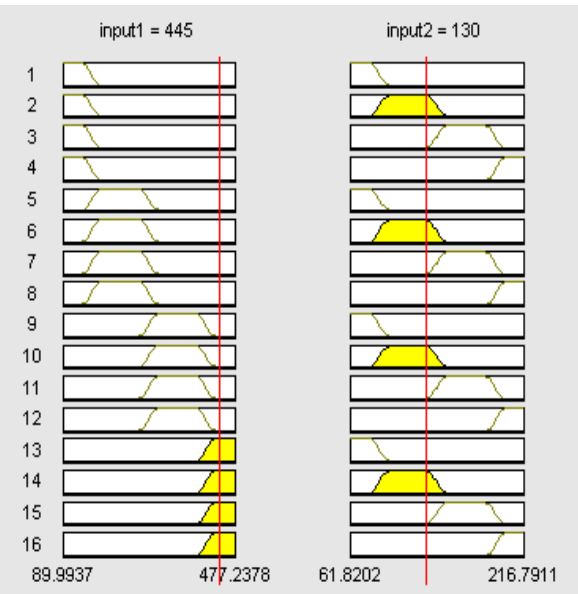

(d) Butterfly[444.98 130.34]

Fig. 8. Four examples of 16 spline-curve-based rules combining of two ANFIS inputs, [average variance]: (a) Ant, (b) Fish, (c) Grasshopper, and (d) Butterfly 


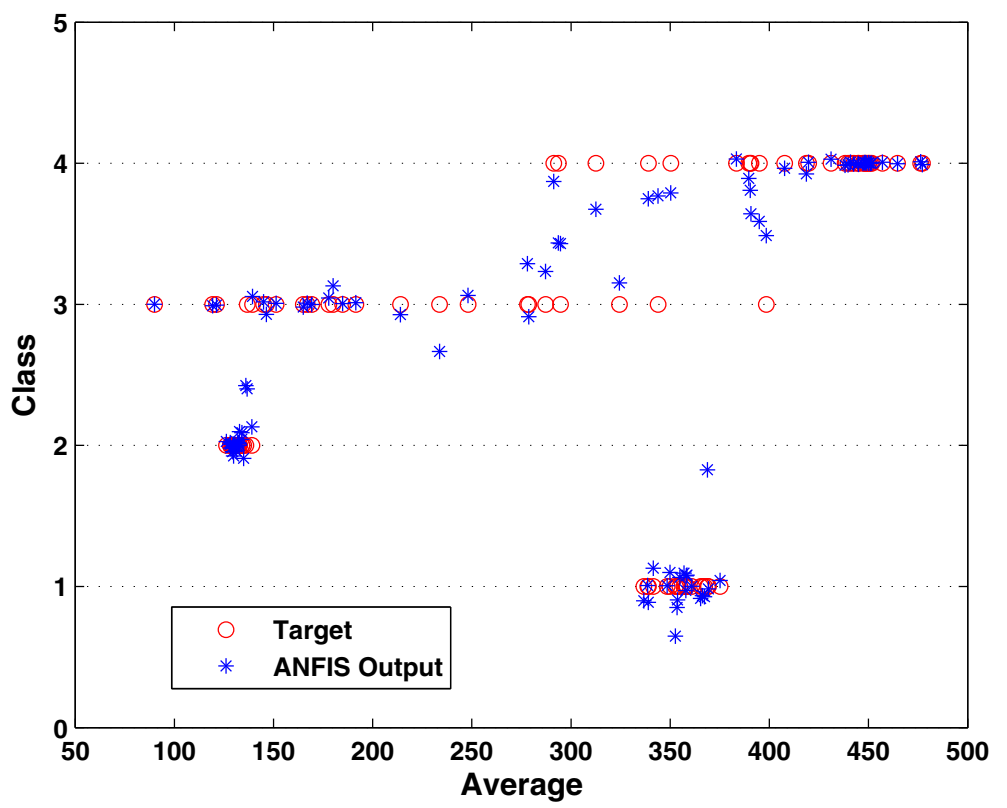

Fig. 9. Training results versus their average of 100 samples

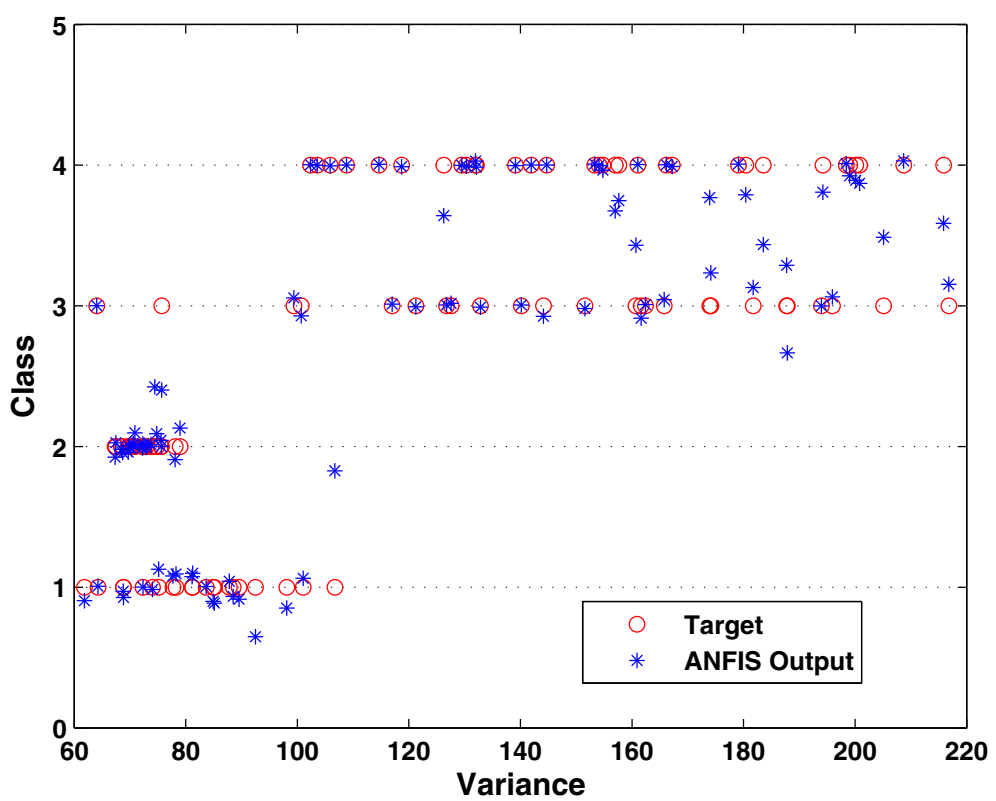

Fig. 10. Training results versus their variance of 100 samples 


\section{Experimental Results}

In our experiment, we generated the total of 100 synthetic data by using the aforementioned procedures. Using the uniform distribution to generates the data portion, the percentages of the four styles are $21,22,25$, and 32 , respectively. In the off-line stage, we used the spline-based curve, i.e., a built-in membership function in MATLAB. Its parameters $a$ and $d$ locate the feet of the curve, while $b$ and $c$ locate its shoulders as shown in Fig. 7. The number of membership functions are four per variable; therefore, four by four rules are produced from two variables, i.e., the average and variance of a visitor's attention. The ANFIS outputs are four classes corresponding to ant, fish, grasshopper, and butterfly styles. In the off-line stage, we trained the ANFIS against the synthetic data and obtained the training error of $4 \%$. Fig. 8 shows 16 produced rules built by spline curves. The degree of color intensity in spline curves indicate the fuzzy degree of the average or variance in according to rules. Fig. 9] and 9 show the ANFIS outputs comparing with their targets versus their averages and their variances, respectively.

The on-line stage is tested against 36 avatars in RDAP (Ritsumeikan Digital Archiving Pavilion). RDAP is a virtual gallery displaying the kimono textiles of 19 objects. All avatars intentionally explored the gallery following their assigned styles. In this experiment, the number of ant, fish, grasshopper, and butterfly visitors are equally set to 9 . Then, the classification accuracy of on-line stage is $83 \%$.

\section{Conclusions and Discussions}

In this paper, we propose the on-line classification system that its off-line stage can be trained by using the synthetic data. We, also, introduce the algorithms of generating the synthetic data of four visiting styles as defined in [510]. We, then, conduct an adaptive neuro-fuzzy inference system (ANFIS) trained by the synthetic visitor data in the off-line stage and tested by the new visitor data in the on-line stage.

Since the overlapping between two clusters of grasshopper and butterfly, we exploit fuzzy paradigm for dealing with continuous and imprecise variables. Two reasons of using ANFIS are the followings. First, fuzzy inference system (FIS) employing fuzzy if-then rules that can model the qualitative aspects of human knowledge and reasoning processes without employing precise quantitative analyses. Second, ANFIS uses a hybrid learning algorithm to identify parameters of Sugeno-type fuzzy inference systems.

In our future work, we will implement the on-line classification in Second Life in order to investigate user behaviors in 3D virtual museums comparing with those in real museums. A recommendation of personal route map is one of applications when a 3D virtual museum can classify the visitor style of a user. The system can recommend new artifacts, exhibitions, and events and navigate a route direction by using our proposed function of measuring the visitors' attention of each exhibit. 


\section{References}

1. Urban, R., Marty, P., Twidale, M.: A second life for your museum: 3d multiuser virtual environments and museums. In: Museums and Web 2007. Archives \& Museum Informatics, Toronto (March 2007)

2. Sookhanaphibarn, K., Thawonmas, R.: A framework for design and evaluation of digital museums in second life as learning institutions. In: Proceedings of International Conference on Information Hiding and Multimedia Signal Processing (IIH-MSP). IEEE, Los Alamitos (2009)

3. Sookhanaphibarn, K., Thawonmas, R.: A content management system for userdriven museums in second life. In: Proceedings of International Conference on Cyberworld. IEEE, Los Alamitos (2009)

4. Oberlander, J., Karakatsiotis, G., Isard, A.: Androutsopoulos: Building an adaptive museum gallery in second life. In: Proceedings of Museum and the Web, Montreal, Quebec, Canada, pp. 749-753 (2008)

5. Veron, E., Levasseur, M.: Bibliothque publique d'Information. Centre Georges Pompidou, Paris (1983)

6. Bianchi, A., Zancanaro, M.: Tracking users' movements in an artistic physical space. In: The $i^{3}$ Annual Conference, held in Siena, Italy, October 20-22, pp. 103 106 (1999)

7. Gabrielli, F., Marti, P., Petroni, L.: The environment as interface. In: The $i^{3}$ Annual Conference, held in Siena, Italy, October 20-22, pp. 44-47 (1999)

8. Zancanaro, M., Kuflik, T., Boger, Z., Goren-Bar, D., Goldwasser, D.: Analyzing museum visitors behavior patterns. In: Conati, C., McCoy, K., Paliouras, G. (eds.) UM 2007. LNCS (LNAI), vol. 4511, pp. 238-246. Springer, Heidelberg (2007)

9. Hatala, M., Wakkary, R.: Ontology-based user modeling in an augmented audio reality system for museums. User Modeling and User-Adapted Interaction (3-4), 339-380 (2005)

10. Chittaro, L., Ieronutti, L.: A visual tool for tracing users' behavior in virtual environments. In: AVI 2004: Proceedings of the Working Conference on Advanced Visual Interfaces, pp. 40-47. ACM, New York (2004)

11. Kotz, S., Balakrishnan, N., Johnson, N.L.: Continuous Multivariate Distributions, Models and Applications, 2nd edn. Wiley Series in Probability and Statistics, vol. 1 (2000)

12. Sookhanaphibarn, K., Thawonmas, R.: A movement data analysis and synthesis tool for museum visitors' behaviors. In: Proceedings of Pacific-Rim Conference on Multimedia. IEEE, Los Alamitos (2009) 